\title{
A Variance Multi-period Portfolio Model with Fixed Ratio Based on Ternary Interval Numbers
}

\author{
Liu Ximei ${ }^{1, *}$, Wang Changfeng ${ }^{1}$ and Lv Yuan $^{2}$ \\ ${ }^{1}$ School of Economics and Management, Beijing University of Posts and \\ Telecommunications, Beijing 100876, China \\ ${ }^{2}$ Information Service Department, Nanjing Tech University, Nanjing 211800 , \\ China \\ 1092457487@qq.com; wangcf@bupt.edu.cn; rarny@163.com
}

\begin{abstract}
In portfolio problem, the expected return, risk etc. cannot be predicted precisely. The investor generally makes his portfolio decision according to his experience. So, deterministic portfolio selection is not a good choice for the investor. Portfolio selection of oil/ gas projects is a fundamental subject of capital budgeting in the energy sector. Interval number is widely used to model the problem in uncertain environments in most of the recent works. In this paper, we combine forecasting and decision-making, and utilize the concept of the ternary interval numbers to build a variance multi-period portfolio optimization model with fixed ratio. In addition, we also give three weak optimal solutions of the proposed model. Finally, these approaches are tested on a set of project data from CNOOC(China National Offshore Oil Corporation).
\end{abstract}

Keywords: Risk; Multi-period; Ternary interval numbers; Optimal allocation

\section{Introduction}

Energy plays an important role in the global economy. The portfolio about oil/ gas projects is also important for the industry. The aim of portfolio selection is to choose a set of projects from a pool of possible projects such that the value of financial actions is maximized on resource availability and technical limitations [1]. Modern portfolio theory offers how to choose a portfolio with feasible expected returns [2]. Then, The mean-variance model [3], multi-period semi-variance model [4], and nonlinear futures hedging model [5] were proposed.

In terms of application, Wang Jun et al. [6] studied the project portfolio model of insurance companies by applying the nonlinear programming method. Hong Bowen et al. [7] constructed a general model of dynamic multi-objective optimal dispatch, and applied the model in the day-ahead optimal dispatch of a typical PV-wind-battery-diesel microgrid. Tomás et al. [8] proposed the application of Markowitz's portfolio theory to the selection of digital communication channel.

In terms of method or algorithm, Cao Juan[9] presented a method based on genetic algorithm multi-objective optimization portfolio planning system. Qi Yutao et al. [10] proposed a memetic immune algorithm for multi-objective optimization by introducing the pareto dominance. Chen Jie et al. [11] adopted the maximum fuzzy satisfaction degree method to transform the multi-objective optimization problem into a non-linear single-objective optimum problem.

In terms of interval model, Chen Guohua et al. [12] proposed a new interval programming model with $\beta$ value. Fan Ting et al. [13] built a linear programming model and derived the optimal decisions for the portfolio. Sun Chong et al. [14] presented a multi-objective interval number linear programming model about the portfolio investment and the given the fuzzy optimization model based on interval number. 
However, existing portfolio selection methods and models do not sig-nificantly consider the dimension of time. Because single-period investors lack of the efficiency of investment decision-making, so we need to build multi-period dynamic models [15]. Mossin [16] and Samuelson [17] extended the single-period model to multi-period model. Chi Guotai et al. [18] established a multi-period dynamic portfolio optimization model using the inverse recursive principle and the nonlinear programming method. Edirisinghe [19] was concerned with an investor trading in multiple securities over many time periods in order to meet an outstanding liability at some future date. Zhang Jinli et al. [20] proposed a discrete-time version of dynamic portfolio selection model for survival in fuzzy environments.

Though a considerable number of research papers has been published for portfolio selection problem, none has considered the variance multi-period portfolio selection model with fixed investment ratio based on ternary interval numbers. The paper is organized as follows. In Section 2, we give a short discussion on ternary interval numbers. In Section 3, we will introduce the variance multi-period portfolio selection model with fixed investment ratio using the concept of ternary interval numbers, then we also give the three weak optimal solutions of the model. In Section 4, an application to the Oil Company is given to illustrate how to get the three weak optimal solutions, this section also includes a discussion of the results. Summary and conclusion is given in Section 5.

\section{Ternary Interval Numbers and Ternary Interval Arithmetic}

Ternary interval is to use 3 parameters to represent a ternary interval, denoted as:

$$
A=[\underline{a}, a, \bar{a}], \underline{a} \leq a \leq \bar{a}
$$

where $\underline{a}$ is the lower bound, $\bar{a}$ is the upper bound of interval, and $a$ is the maximum number of possible values of interval $A$. The center and the width of $A$ are defined as

$$
m(A)=\frac{\underline{a}+\bar{a}}{2} \text { 和 } w(A)=\frac{\bar{a}-\underline{a}}{2}
$$

We extract some fundamental concepts [21], as follows:

Definition1. Let $R$ be the set of all the real numbers. For any two ternary interval numbers $A=[\underline{a}, a, \bar{a}]$ and $B=[\underline{b}, b, \bar{b}]$, there will has:

$$
\begin{gathered}
A+B=B+A=[\underline{a}+\underline{b}, a+b, \bar{a}+\bar{b}] \\
A-B=[\underline{a}-\underline{b}, a-b, \bar{a}-\bar{b}] \\
A \pm k=[\underline{a} \pm k, a \pm k, \bar{a} \pm k], k \in R \\
k A=k[\underline{a}, a, \bar{a}]=\left\{\begin{array}{l}
{[k \underline{a}, k a, k \bar{a}], k \geq 0} \\
{[k \bar{a}, k a, k \underline{a}], k<0}
\end{array}, k \in R\right.
\end{gathered}
$$

\section{The Portfolio Selection Model}

In this section, we will first describe the assumptions and notations used in the construction of the paper. Then the variance multi-period portfolio selection model with fixed investment ratio based on ternary interval numbers will be constructed in the next subsection, and then we also give the three weak optimal solutions of the model . 


\subsection{Assumptions and Notations}

We assume that a decision maker allocates his/her company's wealth among $n(n \geq 2)$ kinds of projects, the initial capital is $W_{0}$, and there are $T$ investment periods. Then we decide how to construct the variance multi-period portfolio selection model with fixed investment ratio based on ternary interval numbers. We introduce some notations as follows:

$n:$ The total number of projects;

$r_{t i}$ : The lower limit of the expected return on project $i$ in period $t$;

$r_{t i}$ : The expected return on the project $i$ in period $t$;

$\bar{r}_{t i}:$ The upper limit of the expected return on project $i$ in period $t$;

$\tilde{r}_{t i}=\left[\underline{r}_{t i}, r_{t i}, \bar{r}_{t i}\right]$ : The ternary interval of the expected return on project $i$ in period $t$; $\underline{v}_{t i}$

: The lower limit of the variance on project $i$ in period $t$;

$v_{t i}$

: The variance on project $i$ in period $t$

_ : The upper limit of the variance on project $i$ in period $t$;

$v_{t i}$

$\tilde{v}_{t i}=\left[\underline{v}_{t i}, v_{t i}, \bar{v}_{t i}\right]$ : The ternary interval of the variance on project $i$ in period $t$;

$x_{t i}$ : The proportion of wealth allocated to project $i$ in period $t$;

$x_{t}=\left(x_{t 1}, x_{t 2}, \cdots, x_{t n}\right)^{T}$ : The $n$-dimensional vector of decision variables in period $t$;

$W_{t}$ : Investors' wealth at the end of period $t-1$ (at the beginning of period $t$ );

$l_{t i}$ : The lower limit of wealth allocated to project $i$ in period $t$;

$\mu_{t i}$ : The upper limit of wealth allocated to project $i$ in period $t$;

The expected return of portfolio $x_{t}=\left(x_{t 1}, x_{t 2}, \cdots, x_{t n}\right)^{T}$ is given by

$\tilde{r}\left(x_{t}\right)=\sum_{i=1}^{n} \tilde{r}_{t i} x_{t i}$

The variance of portfolio $x_{t}=\left(x_{t 1}, x_{t 2}, \cdots, x_{t n}\right)^{T}$ is given by

$$
\tilde{v}\left(x_{t}\right)=\sum_{i=1}^{n} \tilde{v}_{t i} x_{t i}^{2}
$$

We also impose the capital budget constraint in period $t$ as:

$$
\sum_{i=1}^{n} x_{t i}=1, t=1,2, \cdots, T
$$

and the no short-selling constraint as:

$$
x_{t i} \geq 0, i=1,2, \cdots, n, t=1,2, \cdots, T
$$

\subsection{Construction of the Variance Multi-period Portfolio Model with Fixed Investment Ratio based on Ternary Interval Numbers}

Using assumptions and notations introduced in the previous subsection, the ternary interval of expected return in period $t$ of portfolio $x_{t}=\left(x_{t 1}, x_{t 2}, \cdots, x_{t n}\right)^{T}$ is thus given by 


$$
\tilde{r}\left(x_{t}\right)=\sum_{i=1}^{n} \tilde{r}_{t i} x_{t i}=\left[\sum_{i=1}^{n} \underline{r}_{t i} x_{t i}, \sum_{i=1}^{n} r_{t i} x_{t i}, \sum_{i=1}^{n} \bar{r}_{t i} x_{t i}\right]
$$

The ternary interval of the variance in period $t$ of portfolio $x_{t}=\left(x_{t 1}, x_{t 2}, \cdots, x_{t n}\right)^{T}$ is thus given by

$$
\tilde{v}\left(x_{t}\right)=\sum_{i=1}^{n} \tilde{v}_{t i} x_{t i}^{2}=\left[\sum_{i=1}^{n} \underline{v}_{t i} x_{t i}^{2}, \sum_{i=1}^{n} v_{t i} x_{t i}^{2}, \sum_{i=1}^{n} \bar{v}_{t i} x_{t i}^{2}\right]
$$

We can get the relationship between investors' terminal wealth at the end of period $t$ and investors' wealth at the end of period $t-1$ is shown as follows:

$$
W_{t+1}=W_{t}\left(\sum_{i=1}^{n} \tilde{r}_{t i} x_{t i}\right)=W_{t}\left(\sum_{i=1}^{n} \underline{r}_{t i} x_{t i}, \sum_{i=1}^{n} r_{t i} x_{t i}, \sum_{i=1}^{n} \bar{r}_{t i} x_{t i}\right)
$$

As a result, investors' terminal wealth at the end of period $t$ can be expressed as:

$$
W_{T+1}=W_{0} \prod_{t=1}^{T}\left(\sum_{i=1}^{n} \tilde{r}_{t i} x_{t i}\right)=W_{0} \prod_{t=1}^{T}\left(\sum_{i=1}^{n} \underline{r}_{t i} x_{t i}, \sum_{i=1}^{n} r_{t i} x_{t i}, \sum_{i=1}^{n} \bar{r}_{t i} x_{t i}\right)
$$

In order to simplify the presentation, we will note the terminal wealth of the upper, middle and lower bound, respectively, as:

$$
\begin{gathered}
\underline{W}_{T+1}(x)=W_{0} \prod_{t=1}^{T}\left(\sum_{i=1}^{n} \underline{r}_{t i} x_{t i}\right) \\
W_{T+1}(x)=W_{0} \prod_{t=1}^{T}\left(\sum_{i=1}^{n} r_{t i} x_{t i}\right) \\
\bar{W}_{T+1}(x)=W_{0} \prod_{t=1}^{T}\left(\sum_{i=1}^{n} \bar{r}_{t i} x_{t i}\right)
\end{gathered}
$$

Assuming that the investor take risk(variance) as the decision factor, he requires each risk must be less than a given expected level, and he also tries to find a multi-period portfolio strategy to maximize the terminal wealth of the portfolio. In view of these three situations above, the variance multi-period portfolio model with fixed investment ratio based on ternary interval numbers can be formally stated as follows:

$$
\left\{\begin{array}{l}
\max W_{T+1}=\left[\underline{W}_{T+1}(x), W_{T+1}(x), \bar{W}_{T+1}(x)\right] \\
\text { s.t. }\left[\sum_{i=1}^{n} \underline{v}_{t i} x_{t i}^{2}, \sum_{i=1}^{n} v_{t i} x_{t i}^{2}, \sum_{i=1}^{n} \bar{v}_{t i} x_{t i}^{2}\right] \leq v_{t} \\
\sum_{i=1}^{n} x_{t i}=1 \\
0 \leq x_{t i} \leq 1, i=1,2, \cdots, n ; t=1,2, \cdots, T
\end{array}\right.
$$

Where, $v_{t}=\left[v_{t}, v_{t}, \bar{v}_{t}\right]$ is a ternary interval number represents the maximum risk tolerance value in period ${ }^{t}$. We can't find the optimal solution of model (1), but we can find the three weak optimal solutions. The three weak optimal solutions of the model(1) are equal to the optimal solutions of three general programming models (2), (3) and (4)[22]: 


$$
\begin{aligned}
& \left\{\begin{array}{l}
\max \underline{W}_{T+1}(x) \\
\text { s.t. } \sum_{i=1}^{n} \underline{v}_{t i} x_{t i}^{2} \leq \underline{v}_{t} \\
\sum_{i=1}^{n} x_{t i}=1 \\
l_{t i} \leq x_{t i} \leq u_{t i} \\
0 \leq x_{t i} \leq 1, i=1,2, \cdots, n ; t=1,2, \cdots, T
\end{array}\right. \\
& \left\{\begin{array}{l}
\max W_{T+1}(x) \\
s . t \cdot \sum_{i=1}^{n} v_{t i} x_{t i}^{2} \leq v_{t} \\
\sum_{i=1}^{n} x_{t i}=1 \\
l_{t i} \leq x_{t i} \leq u_{t i} \\
0 \leq x_{t i} \leq 1, i=1,2, \cdots, n ; t=1,2, \cdots, T
\end{array}\right. \\
& \left\{\begin{array}{l}
\sum_{i=1}^{n} x_{t i}=1 \\
l_{t i} \leq x_{t i} \leq u_{t i} \\
0 \leq x_{t i} \leq 1, i=1,2, \cdots, n ; t=1,2, \cdots, T \\
s . t . \sum_{i=1}^{n} \bar{v}_{t i} x_{t i}^{2} \leq \bar{v}_{t}
\end{array}\right.
\end{aligned}
$$

\section{Experiment Analysis}

\subsection{Data Description}

We selected four kinds of projects, and the yearly data used in this study is from TE system of CNOOC. The time interval for a period are ten years and there are 3 investment periods. Here, return $=1+($ total profit-income tax $) /$ total income.

\subsection{Determination of Ternary Interval Numbers}

In recent years, many scholars have more research results on portfolio investment model based on ternary interval numbers, but most of them are concentrated in the field of mathematics, less research on practicality and usability. Especially when post-processing the data, the determination of the return or the risk ternary interval, there is no improvement in the applicability of these models. According to the existing literature, this paper selects a reasonable scientific method to determine the return and the risk ternary interval.

Some scholars have suggested that we could use the old return to estimate the future 
return [23]. Since the data selected in this paper is less, so we select grey model to predict the expected return. Academics generally believe that the variance can be used as a measure of risk indicators, and the risk is not a value, but should is a ternary interval number. Then according to the literature[24,25], we get the return and risk ternary interval for 3 investment periods listed in Table1, Table 2 and Table 3.

Table 1. The Return and Risk Ternary Interval in Period 1

\begin{tabular}{lll}
\hline Project & Return $\left(\tilde{r}_{1 i}\right)$ & Variance $\left(\tilde{v}_{1 i}\right)$ \\
\hline Project 1 & {$[1.3314,1.3860,1.4406]$} & {$[0.0029,0.0030,0.0031]$} \\
Project 2 & {$[1.4384,1.4596,1.4808]$} & {$[0.0004,0.0005,0.0006]$} \\
Project 3 & {$[1.1915,1.3147,1.4378]$} & {$[0.0151,0.0152,0.0153]$} \\
Project 4 & {$[0.9152,0.9619,1.0086]$} & {$[0.0021,0.0022,0.0023]$} \\
\hline
\end{tabular}

Table 2. The Return and Risk Ternary Tnterval in Period 2

\begin{tabular}{lll}
\hline Project & Return $\left(\tilde{r}_{2 i}\right)$ & Variance $\left(\tilde{v}_{2 i}\right)$ \\
\hline Project 1 & {$[1.4607,1.4997,1.5386]$} & {$[0.0014,0.0015,0.0016]$} \\
Project 2 & {$[1.4926,1.5051,1.5175]$} & {$[0.0001,0.0002,0.0003]$} \\
Project 3 & {$[1.4121,1.5037,1.5954]$} & {$[0.0083,0.0084,0.0085]$} \\
Project 4 & {$[1.1907,1.2030,1.2152]$} & {$[0.0000,0.0001,0.0002]$} \\
\hline
\end{tabular}

Table 3. The Return and Risk Ternary Interval in Period 3

\begin{tabular}{lll}
\hline Project & Return $\left(\tilde{r}_{3 i}\right)$ & Variance $\left(\tilde{v}_{3 i}\right)$ \\
\hline Project 1 & {$[1.0498,1.1414,1.2329]$} & {$[0.0083,0.0084,0.0085]$} \\
Project 2 & {$[0.8102,0.9841,1.1580]$} & {$[0.0301,0.0302,0.0303]$} \\
Project 3 & {$[1.2872,1.3622,1.4373]$} & {$[0.0055,0.0056,0.0057]$} \\
Project 4 & {$[1.1584,1.1747,1.1911]$} & {$[0.0002,0.0003,0.0004]$} \\
\hline
\end{tabular}

Investment ratio intervals for the four projects are shown in Table4.

Table4. Investment Ratio Intervals for the Four Projects

\begin{tabular}{lllll}
\hline Project & Project 1 & Project 2 & Project 3 & Project 4 \\
\hline Lower Limit & 0.15 & 0.18 & 0.14 & 0.10 \\
Upper Limit & 0.75 & 0.25 & 0.78 & 0.67 \\
\hline
\end{tabular}

\subsection{Result Analysis}

Assuming the initial capital is $W_{0}=10000$, and the maximum risk ternary interval tolerance values of each periods are set to [0.0009,0.0036,0.0062], [0.0010,0.0020,0.0030] and $[0.0016,0.0068,0.0120]$, respectively. For the data we consider, in sight of models (2)-(4) in Section 3.2, and we use LINGO software to solve the three models. The optimal allocations are shown in Table 5. 
Table 5. The Optimal Allocations for Each Model

\begin{tabular}{llllll}
\hline Model & Period t & Project 1 & Project 2 & Project 3 & Project 4 \\
\hline \multirow{3}{*}{$(2)$} & $\mathrm{t}=1$ & 0.4153 & 0.2500 & 0.1400 & 0.1947 \\
& $\mathrm{t}=2$ & 0.5100 & 0.2500 & 0.1400 & 0.1000 \\
& $\mathrm{t}=3$ & 0.1500 & 0.1800 & 0.2719 & 0.3981 \\
\hline \multirow{3}{*}{)} & $\mathrm{t}=1$ & 0.5100 & 0.2500 & 0.1400 & 0.1000 \\
& $\mathrm{t}=2$ & 0.1690 & 0.2500 & 0.4810 & 0.1000 \\
& $\mathrm{t}=3$ & 0.1500 & 0.1800 & 0.5700 & 0.1000 \\
\hline \multirow{3}{*}{)} & $\mathrm{t}=1$ & 0.5100 & 0.2500 & 0.1400 & 0.1000 \\
& $\mathrm{t}=2$ & 0.1500 & 0.1800 & 0.5700 & 0.1000 \\
& $\mathrm{t}=3$ & 0.1500 & 0.1800 & 0.5700 & 0.1000 \\
\hline
\end{tabular}

The optimal allocations graph under the three models is shown in Figure1, Figure1 and Figure3, respectively.

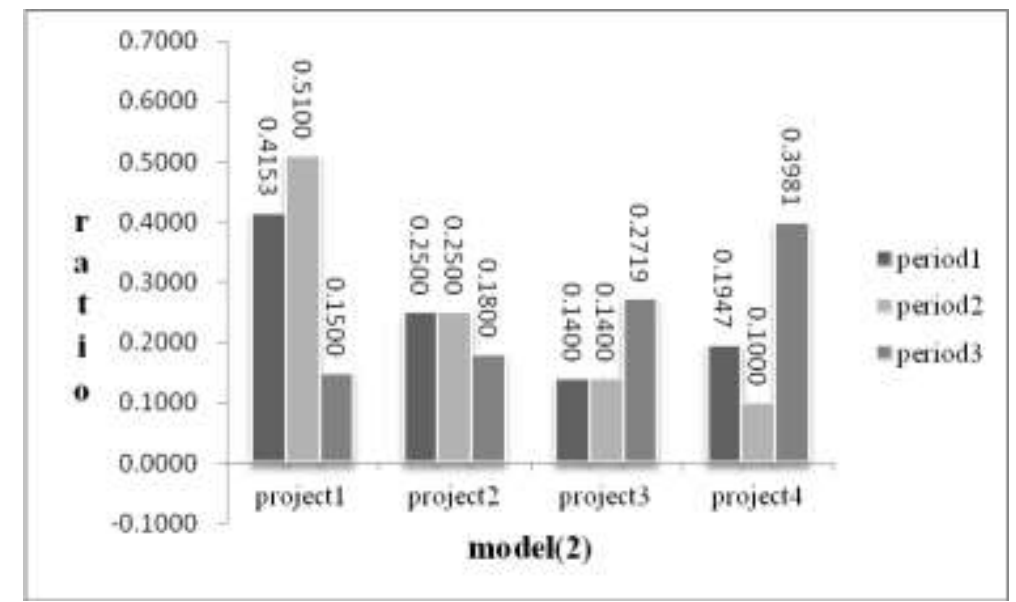

Figure 1. The Optimal Allocations Graph under Model (2)

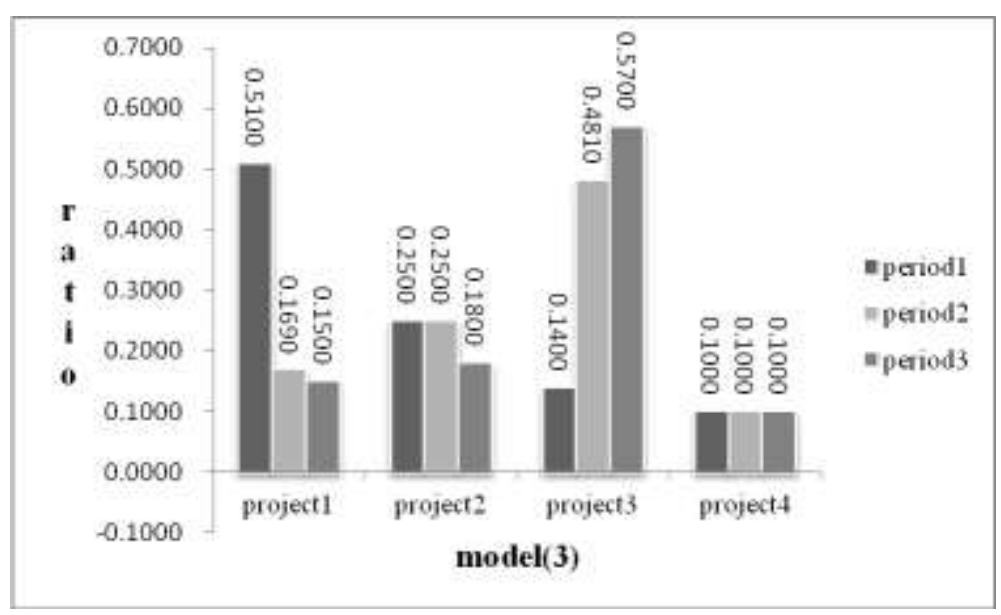

Figure 2. The Optimal Allocations Graph under Model (3) 


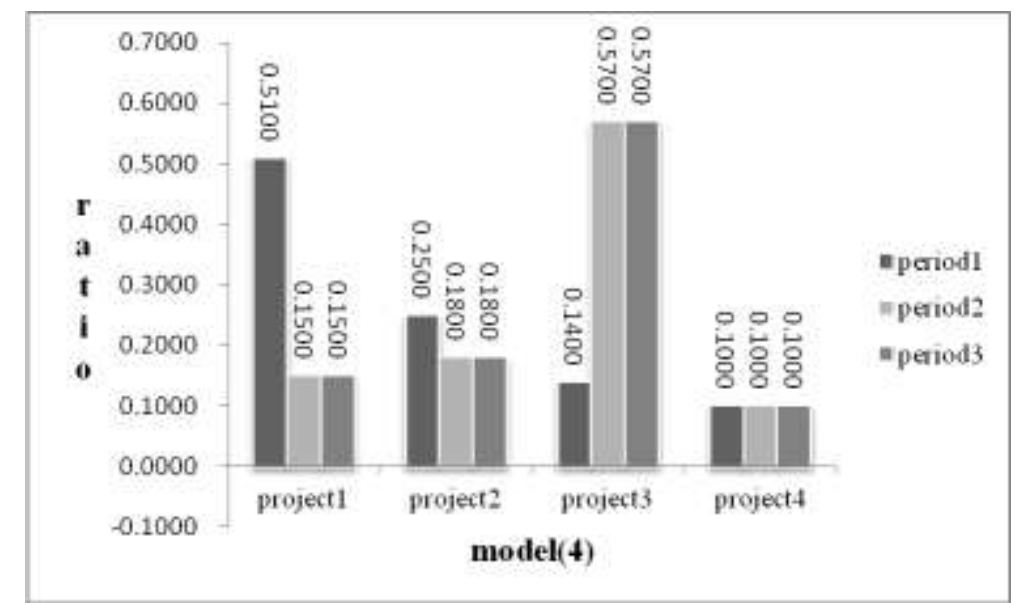

Figure 3. The Optimal Allocations Graph under Model (4)

From Table 5 and Figure 1-3, it is found that the optimums are different at each period.

From Figure 1, we can find that in period 1, the initial wealth ${ }^{W_{0}}$ should be allocated to the four projects with $41.53 \%, 25.00 \%, 14.00 \%$ and $19.47 \%$, respectively. In period 2 , compared with period 1 , the investment ratio of project 1 is increased, project 4 is decreased, and project 2 (project 3) is unchanged, these are mainly due to period 2 is faster than period 1 in respects of return gains and risk reduction of project 1 . In period 3 , compared with period 2, the investment ratio of project 1 and project 2 have reached to the lower limit, this is mainly due to period 3 is faster than period 2 in respects of return reduction of project 1 and project 2 .

From Figure 2, we can find that in period 1, the wealth $W_{1}$ should be allocated to the four projects with $51.00 \%, 25.00 \%, 14.00 \%$ and $10.00 \%$, respectively. In period 2, compared with period 1 , the investment ratio of project 1 is decreased, project 3 is increased, and project 2 (project 4 ) is unchanged, these are mainly due to period 3 is faster than period 1 in respect of return gains of project 3 . In period 3, compared with period 2, the investment ratio of project 1, project 2 and project 4 have reached to the lower limit, this is mainly due to period 3 is faster than period 2 in respects of return reduction of project 1 and project 2 .

From Figure 3, we can find that in period 1, the wealth $W_{2}$ should be allocated to the four projects with $51.00 \%, 25.00 \%, 14.00 \%$ and $10.00 \%$, respectively. In period 2 , compared with period 1, the investment ratio of project 1 is decreased, project 3 is increased, and project 2 (project 4 ) is unchanged, these are mainly due to period 3 is faster than period 1 in respect of return gains of project 3 . In period 3, compared with period 2, the investment ratio of project 1 , project 2 and project 4 have reached to the lower limit, this is mainly due to period 3 is slower than period 2 in respects of return gains and risk reduction of project 3 .

According to the data in Table 5, we can get the risk ternary interval and terminal wealth ternary interval of each model shown in Table 6 . In order to compare the three models, we use the mean of the terminal wealth ternary interval to represent the investor's expected terminal wealth value. 
Table 6. The Risk Interval and Terminal Wealth for Each Model

\begin{tabular}{|c|c|c|c|c|}
\hline $\begin{array}{l}\text { Mode } \\
1\end{array}$ & $\begin{array}{l}\text { Period } \\
t\end{array}$ & Risk Interval & Wealth Interval & $\begin{array}{l}\text { Wealth } \\
\text { Value }\end{array}$ \\
\hline (2) & $\begin{array}{l}t=1 \\
t=2 \\
t=3\end{array}$ & $\begin{array}{l}{[0.0009,0.0009,0.001} \\
0] \\
{[0.0005,0.0006,0.000} \\
6] \\
{[0.0016,0.0016,0.001} \\
7]\end{array}$ & $\begin{array}{l}{[20109,22909,2594} \\
0]\end{array}$ & 22986 \\
\hline (3) & $\begin{array}{l}t=1 \\
t=2 \\
t=3\end{array}$ & $\begin{array}{l}{[0.0011,0.0011,0.001} \\
2] \\
{[0.0020,0.0020,0.002} \\
0] \\
{[0.0030,0.0030,0.003} \\
0]\end{array}$ & $\begin{array}{l}{[21206,24745,2863} \\
8]\end{array}$ & 24863 \\
\hline (4) & $\begin{array}{l}t=1 \\
t=2 \\
t=3\end{array}$ & $\begin{array}{l}{[0.0011,0.0011,0.001} \\
2] \\
{[0.0027,0.0028,0.002} \\
8] \\
{[0.0030,0.0030,0.003} \\
0]\end{array}$ & $\begin{array}{l}{[21108,24745,2876} \\
0]\end{array}$ & 24871 \\
\hline
\end{tabular}

From Table 6, the terminal wealth ternary interval of model (2) is [20109,22909,25940], the terminal wealth value of model (2) is $W_{(2)}=22986$.The terminal wealth ternary interval of model (3) is [21206,24745,28638], the terminal wealth value of model (3) is $W_{(3)}=24863$.The terminal wealth ternary interval of model (4) is [21108,24745,28760], the terminal wealth value of model (4) is $W_{(4)}=24871$. So, we can get the relationship $W_{(2)}<W_{(3)}<W_{(4)}$.

\section{Conclusion}

We combine forecasting and decision-making and utilize the concept of the ternary interval numbers to introduce a new framework of multi-period portfolio optimization model with fixed investment ratio considering risk. The portfolio risk was characterized by variance. Then three different models have been used to solve the proposed model, and we can get three weak optimal solutions by solving the three different models. Finally, these models are tested on a set of project data from Oil Company, we use LINGO7.0 to solve these models. These results show that the models are reasonable, the optimums are different at each period, and the models presented make investment more flexible. 


\section{References}

[1] A. Arasteh, A. Aliahmadi and M. M. Omran, "A Multi-stage Multi Criteria Model for Portfolio Management”, Arab. J. Sci. Eng, vol. 39, no. 2, (2014), pp. 4269-4283.

[2] M. Gunasekaran, K. S. Ramaswami and S. Karthik, "Integration of SRA, ANFIS and CAPM for Stock Portfolio Management”, Original Research, vol. 1, no. 4, (2013), pp. 291-300.

[3] Wu Xiaoli and Liu Yankui, "Portfolio Selection", J Appl Math Comput, vol. 36, (2011), pp. 373-400.

[4] W. Yan and S. Li , "A Class of Multi-period Semi-variance Portfolio Selection with a Four-factor Futures Price Model”, J. Appl. Math. Comput, vol. 29, (2009), pp. 19-34.

[5] F. Junhui, Z. Weiguo, L. Qian and M. Qin, "Nonlinear Futures Hedging Model based on Skewness Risk and Kurtosis Risk", Systems Engineering, vol. 37, no. 10, (2009), pp. 44-48.

[6] W. Jun and W. Dong, "Research on Optimal Investment Portfolio Proportion of Insurance Companies", Insurance Studies, no. 12, (2010), pp. 60-67.

[7] H. Bowen, G. Li, W. Chengshan and J. Bingqi, "Model and Method of Dynamic Multi-objective Optimal Dispatch for Microgrid”, Electric Power Automation Equipment, vol. 33, no. 3, (2013), pp. 100-107.

[8] T. Frausto-da-Silva, A. Grilo and V. C. Machado, "Selection of Digital Marketing Channels: Application of Modern Portfolio Theory", Industrial Engineering, (2015), pp. 585-596.

[9] C. Juan, "Portfolio Optimization Algorithm for Multi Objective Programming", Xian, Xi'an Shiyou University, (2012), pp. 16-42.

[10] Q. Yutao, L. Fang, C. Weiyuan, M. Xiaoliang and J. Licheng, "Memetic Immune Algorithm for Multi-objective Optimization", Journal of Software, vol. 24, no. 7, (2013), pp. 1529-1544.

[11] C. Jie, Y. Xiu, Z. Lan, Z. Meixia and L. Zhenkun, "Microgrid Multi-objective Economic Dispatch Optimization", Proceedings of the CSEE, vol. 23, no. 19, (2013), pp. 57-66.

[12] C. Guohua, Y. Zhuanjun and L. Xiaolian, "Interval Portfolio Selection Model based on $\beta$ Constraints", Mathematics Economics, vol. 25, no. 3, (2008), pp. 254-256.

[13] F. Ting, Z. Ling and H. Zhongcheng, "Model for Portfolio Selection based on Interval Number Return Rates", Journal of Guilin University of Electronic Technology, vol. 32, no. 2, (2012), pp.167-169.

[14] S. Chong and H. Weibo, "Interval Number-based Portfolio Investment Fuzzy Optimization Model", Journal of Huaibei Normal University (Natural Science), vol. 36, no. 3, (2015), pp.1-4.

[15] T. Sen, "The Study of Log-optimal Portfolio Model under VaR and CVaR Risk", Shanxi, Northwestern Polytechnical University, (2004), pp.1-3.

[16] J. Mossion, "Optimal Multiperiod Portfolio Policies", Journal of Business, vol. 41, (2004), pp. 215-229.

[17] P. A. Samuelson, "Lifetime Portfolio Selection by Dynamic Stochastic Programming", Review of Economics and Statistics, vol. 51, (1969), pp. 239-246.

[18] C. Guotai, D. Hechao and S. Xiuyan, "A Decision-making Model of Bank's Asset Portfolio based on Multi-period Dynamic Optimization”, Systems Engineering-Theory \& Practice, no. 2, (2007), pp.1-16.

[19] N.C.P. Edirisinghe, "Multiperiod Portfolio Optimization with Terminal Liability: Bounds for the Convex Case", Computational Optimization and Applications, vol. 32, (2005), pp. 29-59.

[20] Z. Jinli, T. Wansheng, W. Cheng and Z. Ruiqing, "Fuzzy Dynamic Portfolio Selection for Survival”, LNCS 4681, (2007), pp.4-45.

[21] X. Le, "Decision Information Model and its Applications based on Ternary Interval Number", Anhui, Anhui University, (2012), pp. 5-7.

[22] H. Hua, S. Yanping and D. Turehan, "Research on Interval Numbers of Three Elements Linear Programming and its Solution", Mathematics in Practice and Theory, vol. 41, no. 9, (2011), pp. 134-141.

[23] L. Xuefa and Y. Gujun, "An Empirical Study on Performance Evaluation of Securities Investment Funds", Nankai Economic Studies, no. 5, (2004), pp. 79-84.

[24] L. Tingting, "The Empirical Study and Analysis of Portfolio Model based on Interval Number", Anhui, Anhui University, (2010), pp. 24-33.

[25] L. Xiaoling, "Measurement Method for Stock Investment Expected Return and Risk", Statistics \& Decision, vol. 4, (2005), pp. 30-32. 


\section{Authors}

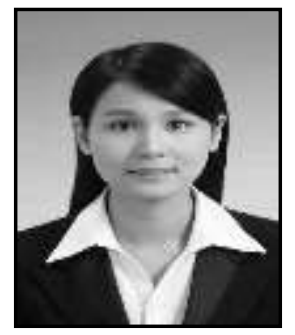

Liu Ximei, Miss. Liu Ximei is a Ph.D. researcher at Beijing University of Posts and Telecommunications, China. She holds post-graduation qualifications in probability and mathematical statistics. Her areas of interests are forecasting, risk control, and portfolio management.

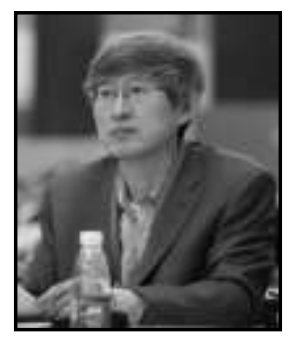

Wang ChangFeng, Prof. Dr. Wang ChangFeng is the director of the International Project Management Institute in Beijing University of Posts and Telecommunications, professor and Ph.D. supervisor. He is an expert in guiding the PMI GAC project management accreditation, assessment expert for PMI GAC CRC project management accreditation. His main research areas include the enterprise project management of safety risk early warning and emergency response, and the complex system integration and control of major projects and programs.

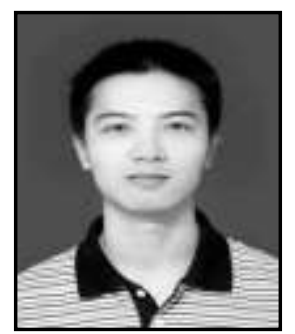

Lv Yuan, Mr. Lv Yuan is an administrative staff of Nanjing Tech University, China. Meanwhile, he is also a Ph.D. researcher at Nanjing University, China. He holds post-graduation qualifications in applied mathematics. His areas of interests are big data science and rich web development. 
International Journal of Hybrid Information Technology

Vol. 10, No. 6 (2017) 